\title{
Impact on Signal Integrity of Interconnect Variabilities
}

\author{
Paolo Manfredi*, Dries Vande Ginste ${ }^{\dagger}$, Daniël De Zutter ${ }^{\dagger}$, and Flavio G. Canavero* \\ *EMC Group, Department of Electronics and Telecommunications, Politecnico di Torino \\ Corso Duca degli Abruzzi 24, 10129 Torino, Italy \\ E-mail: paolo.manfredi@polito.it \\ $\dagger$ Electromagnetics Group, Department of Information Technology, Ghent University \\ Sint-Pietersnieuwstraat 41, 9000 Gent, Belgium
}

\begin{abstract}
In this paper, literature results on the statistical simulation of lossy and dispersive interconnect networks with uncertain physical properties are extended to general nonlinear circuits. The approach is based on the expansion of circuit voltages and currents into polynomial chaos approximations. The derivation of deterministic circuit equivalents for nonlinear components allows to retrieve the unknown expansion coefficients with a single circuit simulation, that can be carried out via standard SPICE-type solvers. These coefficients provide direct statistical information. The methodology allows the inclusion of arbitrary nonlinear elements and is validated via transmissionline networks terminated by diodes and driven by inverters.

Index Terms-Circuit simulation, nonlinear, polynomial chaos, signal integrity, SPICE, statistical Analysis, transmission lines.
\end{abstract}

\section{INTRODUCTION}

Signal integrity is becoming increasingly affected by random variations in the physical interconnect properties. As technology scales down, manufacturing tolerances introduce significant variability in the geometry of printed circuit board (PCB) interconnects. Standard circuit simulators usually offer sampling-based (Monte Carlo-like) statistical methods that, however, require a large number of simulation samples, thus often being computationally prohibitive.

Alternative simulation strategies were presented [1]-[4] that are based on the so-called polynomial chaos (PC) framework [5]. According to PC, stochastic voltages and currents are expressed as expansions of orthogonal polynomials, whose coefficients directly provide statistical information. The technique was first applied to the frequency-domain simulation of on-board interconnects [1]. On the one hand, the derivation of SPICE-compatible equivalent circuits allowed the time-domain analysis of arbitrary lossy and dispersive interconnect topologies [2], but still limited to linear circuits. On the other hand, the development of a general framework for the inclusion of arbitrary nonlinear terminations [3] enabled the analysis of structures that include nonlinear drivers and terminations, but the implementation was limited to lossless and dispersion-free lines. Finally, a SPICE integration of the nonlinear device models has been recently presented [4], easing the simulation of general nonlinear circuits. In this paper, the aforementioned approaches [2]-[4] are reviewed, combined and applied to the analysis of lossy interconnect networks with nonlinear sources and terminations.

\section{The Polynomial Chaos Approach}

For the sake of simplicity, the method is introduced by means of a single line and a single random variable (RV). Fig. 1 illustrates an interconnect section of length $\mathcal{L}$, described by the pertinent per-unit-length (p.u.l.) resistance $R$, inductance $L$, conductance $G$ and capacitance $C$, possibly dependent on the angular simulation frequency $\omega$. The line is driven by an inverter and terminated by a diode. Generalization to multiconductor lines and multiple RVs is straightforward [2], [3].

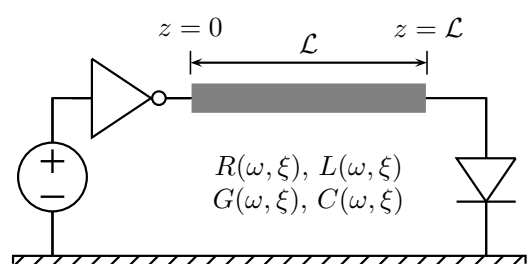

Fig. 1. Pictorial illustration of an interconnect section of length $\mathcal{L}$, with nonlinear source and termination.

It is convenient to express the random parameter via a normalized RV. For example, a Gaussian random parameter $x$ (e.g., the width of a PCB line) with average $\bar{x}$ and standard deviation $\sigma_{x}$, is written as $x=\bar{x}+\sigma_{x} \xi$, where $\xi$ is a standard normal RV with zero mean and unit variance, i.e. $\xi \sim \mathcal{N}(0,1)$. If an interconnect property is random, the p.u.l. parameters become random themselves and $\xi$-dependent (e.g., $R=R(\xi)$ ).

The signal propagation along the coupled lines is described by the Telegraphers' equations [6]:

$$
\begin{gathered}
\frac{d}{d z} \hat{V}(z, \omega, \xi)=-[R(\omega, \xi)+j \omega L(\omega, \xi)] \hat{I}(z, \omega, \xi), \\
\frac{d}{d z} \hat{I}(z, \omega, \xi)=-[G(\omega, \xi)+j \omega C(\omega, \xi)] \hat{V}(z, \omega, \xi),
\end{gathered}
$$

where $\hat{V}$ and $\hat{I}$ are the phasors of the voltage and the current along the line, respectively. They are also $\xi$-dependent, since the uncertain interconnect properties render the circuit response nondeterministic. In SPICE-type simulators, these equations are combined with the governing equations of classical linear elements, like resistors and capacitors, and nonlinear components via the Kirchhoff current law (KCL), 
and then solved in time domain via convolution techniques (see, e.g., [7]).

The traditional approach to tackle stochastic circuits and extract pertinent statistical information is to collect many samples of the circuit response for different values of the random parameters. However, when the circuit size grows, this technique rapidly becomes computationally intractable.

\section{A. The Polynomial Chaos Expansion}

The underlying idea of PC is to express stochastic circuit voltages $v$ and currents $i$ as polynomial expansions, i.e.

$$
v(t, \xi) \approx \sum_{k=0}^{P} v_{k}(t) \varphi_{k}(\xi), \quad i(t, \xi) \approx \sum_{k=0}^{P} i_{k}(t) \varphi_{k}(\xi),
$$

where the basis functions $\varphi_{k}$ are polynomials satisfying the orthonormality condition $\left\langle\varphi_{k}, \varphi_{j}\right\rangle=\delta_{k j}$ (Kronecker's delta) with respect to the following inner product:

$$
\langle f, g\rangle=\int_{\mathbb{R}} f(\xi) g(\xi) w(\xi) d \xi
$$

with $w(\xi)$ the probability density function (PDF) of $\xi$ [5].

The (deterministic) coefficients $v_{k}(t)$ and $i_{k}(t)$ become the new circuit unknowns to be determined. These coefficients provide statistical information. For example, the average voltage corresponds to $v_{0}(t)$, whilst the variance is given by $\sum_{k=1}^{P} v_{k}^{2}(t)$. Similar properties hold for the circuit currents.

\section{B. The Stochastic Galerkin Method}

The strategy for the determination of the unknown PC coefficients is to introduce the PC expansions into the stochastic governing equations and to manipulate them in order to derive a new set of deterministic equations in these coefficients. This task is accomplished by means of a stochastic Galerkin method (SGM) [5] and is illustrated below for (1).

First, the stochastic p.u.l. parameters are also expressed as PC expansions (e.g., $R(\omega, \xi) \approx \sum_{k=0}^{P} R_{k}(\omega) \varphi_{k}(\xi)$, with $R_{k}$ known). Substitution of the PC expansions of the voltages, currents, and p.u.l. parameters into (1), yields:

$$
\begin{aligned}
& \sum_{k=0}^{P} \frac{d}{d z} \hat{V}_{k}(z, \omega) \varphi_{k}(\xi)= \\
& -\sum_{k=0}^{P} \sum_{j=0}^{P}\left[R_{k}(\omega)+j \omega L_{k}(\omega)\right] \hat{I}_{j}(z, \omega) \varphi_{k}(\xi) \varphi_{j}(\xi), \\
& \sum_{k=0}^{P} \frac{d}{d z} \hat{I}_{k}(z, \omega) \varphi_{k}(\xi)= \\
& -\sum_{k=0}^{P} \sum_{j=0}^{P}\left[G_{k}(\omega)+j \omega C_{k}(\omega)\right] \hat{V}_{j}(z, \omega) \varphi_{k}(\xi) \varphi_{j}(\xi),
\end{aligned}
$$

where $\hat{V}_{k}$ and $\hat{I}_{k}$ are the phasors of the voltage and current $\mathrm{PC}$ coefficients, respectively.
In the SGM, the obtained equations (4) are now weighted with the same polynomial basis $\left\{\varphi_{m}\right\}_{m=0}^{P}$ and using the inner product (3):

$$
\begin{aligned}
& \sum_{k=0}^{P} \frac{d}{d z} \hat{V}_{k}(z, \omega)\left\langle\varphi_{k}, \varphi_{m}\right\rangle=\frac{d}{d z} \hat{V}_{m}(z, \omega)= \\
& -\sum_{k=0}^{P} \sum_{j=0}^{P}\left[R_{k}(\omega)+j \omega L_{k}(\omega)\right] \hat{I}_{j}(z, \omega) \alpha_{k j m} \\
& \sum_{k=0}^{P} \frac{d}{d z} \hat{I}_{k}(z, \omega)\left\langle\varphi_{k}, \varphi_{m}\right\rangle=\frac{d}{d z} \hat{I}_{m}(z, \omega)= \\
& -\sum_{k=0}^{P} \sum_{j=0}^{P}\left[G_{k}(\omega)+j \omega C_{k}(\omega)\right] \hat{V}_{j}(z, \omega) \alpha_{k j m}
\end{aligned}
$$

where we defined $\alpha_{k j m}=\left\langle\varphi_{k} \varphi_{j}, \varphi_{m}\right\rangle$ and we used the orthonormality condition in the left-hand sides. It should be noted that $\alpha_{k j m}$ is a deterministic coefficient that can be analytically computed and that the variability vanished since $\xi$ was integrated out.

Equations (5) are still in the form of transmission-line equations like (1). If $m$ is swept from 0 to $P$, a set of $P+1$ coupled deterministic equations is obtained, that can be expressed in matrix form as [2]

$$
\begin{aligned}
& \frac{d}{d z} \hat{\tilde{\mathbf{V}}}(z, \omega)=-[\widetilde{\mathbf{R}}(\omega)+j \omega \widetilde{\mathbf{L}}(\omega)] \hat{\widetilde{\mathbf{I}}}(z, \omega), \\
& \frac{d}{d z} \hat{\widetilde{\mathbf{I}}}(z, \omega)=-[\widetilde{\mathbf{G}}(\omega)+j \omega \widetilde{\mathbf{C}}(\omega)] \hat{\tilde{\mathbf{V}}}(z, \omega),
\end{aligned}
$$

where $\hat{\tilde{\mathbf{V}}}=\left[\hat{V}_{0}, \ldots, \hat{V}_{P}\right]^{T}$ collects all the phasor voltage coefficients, and similarly for $\hat{\widetilde{\mathbf{I}}}$. The new $(P+1) \times(P+1)$ p.u.l. matrices have entries given by

$$
\widetilde{\mathbf{R}}_{m j}(\omega)=\sum_{k=0}^{P} R_{k}(\omega) \alpha_{k j m}
$$

and similarly for $\widetilde{\mathbf{L}}, \widetilde{\mathbf{G}}$ and $\widetilde{\mathbf{C}}$.

In summary, the SGM allows to transform the original, stochastic system of equations (1) into a larger but deterministic one, i.e. (6), governing the behavior of the PC voltage and current coefficients. The simulation of the new equations (6) can be carried out as an augmented transmission line using standard (e.g., SPICE-type) design software, provided that the expansion coefficients $R_{k}, L_{k}, G_{k}, C_{k}$ of the p.u.l. matrices, which are necessary for the determination of the corresponding augmented p.u.l. matrices $\widetilde{\mathbf{R}}, \widetilde{\mathbf{L}}, \widetilde{\mathbf{G}}$ and $\widetilde{\mathbf{C}}$, are calculated [2]. However, the simulation of a complete electrical network requires the derivation of similar equations for the other circuit components. This is discussed in the next section.

\section{Terminal Conditions}

The terminal (boundary) conditions for the solution of the differential equations (6) evolve from the governing equations of the components connected to the line terminations. For the sake of brevity, we limit ourselves to the case of deterministic 
terminations (i.e., the variability affects only the transmissionline elements). Stochastic lumped components, both linear and nonlinear, can also be accounted for [4].

Let us consider the diode at the far-end termination $(z=\mathcal{L})$ in Fig. 1, and assume its I-V relationship is described by a generic operator $\mathcal{F}(\cdot)$. For our purposes, $\mathcal{F}(\cdot)$ may be both an analytical function or a non-explicit characteristic, such as a complex library model. The terminal equation becomes:

$$
i(\mathcal{L}, t, \xi)=\mathcal{F}(v(\mathcal{L}, t, \xi)),
$$

where the time-domain line voltage and current are considered.

Replacing the stochastic variables with their PC expansions leads to

$$
\sum_{k=0}^{P} i_{k}(\mathcal{L}, t) \varphi_{k}(\xi)=\mathcal{F}\left(\sum_{k=0}^{P} v_{k}(\mathcal{L}, t) \varphi_{k}(\xi)\right) .
$$

Application of the SGM produces

$$
\begin{aligned}
& i_{m}(\mathcal{L}, t)=\left\langle\mathcal{F}\left(\sum_{k=0}^{P} v_{k}(\mathcal{L}, t) \varphi_{k}\right), \varphi_{m}\right\rangle \\
& =\int_{\mathbb{R}} \mathcal{F}\left(\sum_{k=0}^{P} v_{k}(\mathcal{L}, t) \varphi_{k}(\xi)\right) \varphi_{m}(\xi) w(\xi) d \xi .
\end{aligned}
$$

Therefore, (10) represents the new terminal condition for the $m$ th termination of the augmented line. However, it is important to note that, the operator $\mathcal{F}(\cdot)$ being nonlinear, the polynomial properties can no longer be used to resolve the integration in the right-hand side, thus making (10) of little use in its present form.

The proposed methodology is to discretize the integral using a quadrature rule [3]. Given a set $\left\{\xi_{q}\right\}$ of $Q$ quadrature nodes, (10) becomes

$$
i_{m}(\mathcal{L}, t) \approx \sum_{q=1}^{Q} \mathcal{F}\left(\sum_{k=0}^{P} v_{k}(\mathcal{L}, t) \varphi_{k}\left(\xi_{q}\right)\right) \varphi_{m}\left(\xi_{q}\right) w_{q}
$$

with $w_{q}$ being the weights corresponding to the nodes $\left\{\xi_{q}\right\}$. It is worth noting that (11) is fully deterministic, since the factors $\varphi_{k}\left(\xi_{q}\right)$ and $\varphi_{m}\left(\xi_{q}\right)$ are merely (precomputable) numbers that correspond to the value of the polynomials calculated at $\xi_{q}$. Although (11) involves an approximation, very high accuracy with a limited number of nodes $Q$ can be achieved by means of Gaussian quadratures [3], [4]. Similar boundary conditions are derived for the near-end (i.e., $z=0$ ) terminations.

\section{SPICE Implementation of a Diode Termination}

In order to allow for the simulation of (11) via SPICEtype software, an equivalent circuit interpretation is required. This is provided in [4] and illustrated in Fig. 2 for the $m$ th termination. For ease of notation, with respect to (11), we defined $a_{k q}=\varphi_{k}\left(\xi_{q}\right), b_{m q}=\varphi_{m}\left(\xi_{q}\right) w_{q}$ and $j_{q}(t)=$ $\mathcal{F}\left(\sum_{k=0}^{P} v_{k}(\mathcal{L}, t) a_{k q}\right)$. The terminal current is a weighted combination of $Q$ diode currents $j_{q}=\mathcal{F}(\cdot)$, which are in turn obtained by applying a voltage to the diode that is a weighed combination of the terminal voltages of the augmented line. These combinations are obtained using dependent sources, as shown in Fig. 2. The diode currents $j_{q}$ are sampled by means of $Q$ auxiliary subcircuits like the one shown in Fig. 2(b). The diode appearing in the subcircuit is the same as in the original circuit. It is relevant to point out that the only difference between the models when $m$ varies resides in the coefficient $b_{m q}$ appearing in the dependent current source in Fig. 2(a).

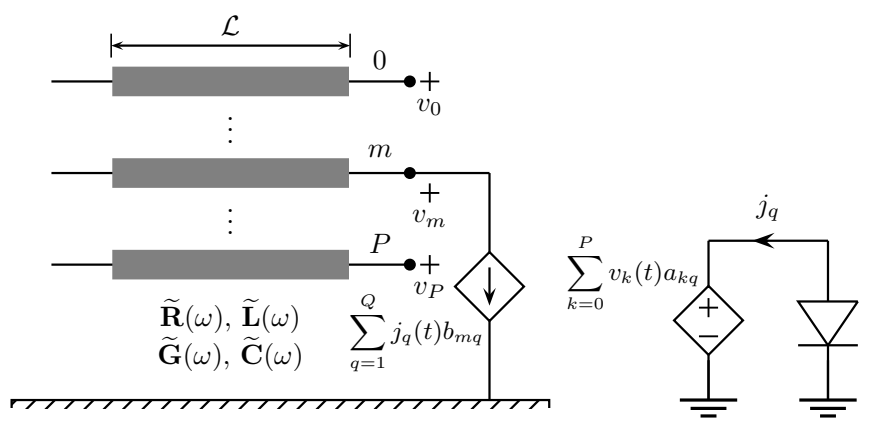

(a)

(b)

Fig. 2. Circuit implementation of a diode termination: equivalent circuit for the $m$ th augmented termination (a) and auxiliary subcircuit that samples the $q$ th diode current $j_{q}$ (b).

\section{E. Modeling of an Inverter}

An analogous approach can be used to model three terminal devices like transistors [4]. For example, Fig. 3 illustrates the modeling of a CMOS inverter. Specifically, Fig. 3(a) shows the original circuit configuration, with p- and n-MOS transistors. In Fig. 3(b), the equivalent circuit for the $m$ th augmented termination is depicted. It should be noted that two dependent current sources are used to model a three-terminal device. These sources suitably combine $\mathrm{p}$ - and n-MOS drain and gate currents sampled by the auxiliary subcircuits in Fig. 3(c) and Fig. 3(d), where the following voltages have been defined:

$$
\begin{aligned}
v_{G S, k}^{n} & =v_{\mathrm{In}, k} \\
v_{D S, k}^{n} & =v_{\mathrm{Out}, k} \\
v_{G S, k}^{p} & =v_{\mathrm{Out}, k}-v_{\mathrm{DD}, k} \\
v_{D S, k}^{p} & =v_{\mathrm{In}, k}-v_{\mathrm{DD}, k}
\end{aligned}
$$

The terminal DD, $m$ is to be properly connected to the power supply.

\section{F. Stochastic Simulation of an Arbitrary Interconnect Network}

For the sake of simplicity, so far we focused the discussion on a single transmission-line segment with a source and a load termination. However, the outlined approach is readily extended to a network of arbitrary complexity. An augmented and deterministic equivalent network is created by replacing each stochastic transmission line with its $(P+1)$-augmented counterpart described by the p.u.l. matrices $\widetilde{\mathbf{R}}, \widetilde{\mathbf{L}}, \widetilde{\mathbf{G}}$, and $\widetilde{\mathbf{C}}$. These transmission-line sections are connected via the equivalent models of lumped components, which in the case of diodes and inverters correspond to the circuits in Figs. 2 and 3 , respectively. It suffices to properly connect each line 


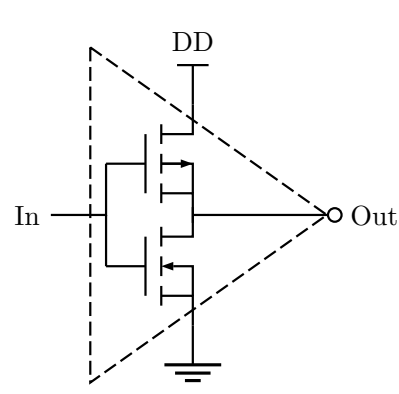

(a)

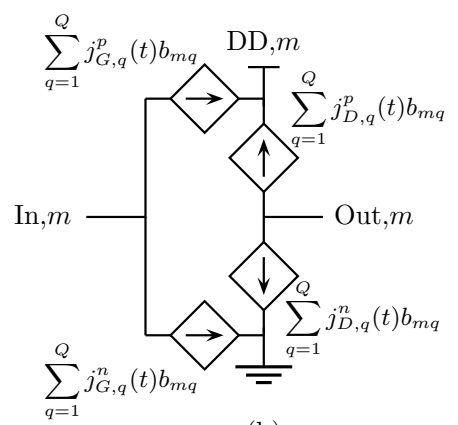

(b)

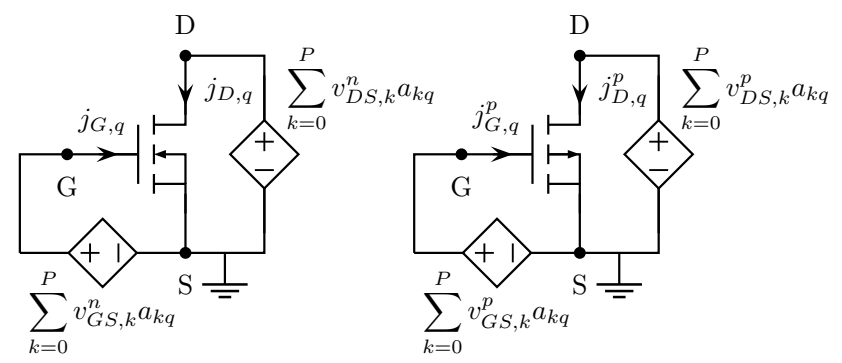

(c)

(d)

Fig. 3. Circuit implementation of an inverter termination: (a) original CMOS configuration; (b) equivalent circuit for the $m$ th augmented termination; (c) circuit that samples the $q$ th $\mathrm{n}$-MOS drain and gate currents; (d) circuit that samples the p-MOS currents.

termination $m$ using the corresponding $m$ th model. On the other hand, linear lumped elements are simply replicated on each termination. Finally, independent sources are kept only on the zeroth termination: all the remaining terminations are in fact shorted when connected to an independent source [2], [4]. The voltages and currents at the terminations of the created augmented network correspond to the PC coefficients of the voltages and currents in the original line. These PC coefficients are then obtained with a single circuit simulation.

\section{Validation and Numerical Results}

This section illustrates the benefits and strength of the advocated technique by means of two application examples, consisting of lossy and dispersive PCB interconnect networks with nonlinear terminations. All the simulations are carried out in HSPICE [7] on an ASUS U30S laptop with an Intel(R) Core(TM) i3-2330M and with CPU running at $2.20 \mathrm{GHz}$ and 4 GB of RAM. Reference results are generated via a Monte Carlo analysis with 1000 samples. The reader is referred to [3], [4] for a comprehensive discussion about the accuracy and convergence properties of both Monte Carlo and PC-based circuit simulations.

\section{A. Example \#1}

The first example considers the interconnect network of Fig. 4. It consists of seven transmission-line sections connected via lumped components and driven by an inverter. All the far-end terminations contain diodes. The transmission lines have a microstrip geometry, also shown in Fig. 4, with copper traces (conductivity: $58 \mathrm{MS} / \mathrm{m}$ ). All the relevant geometric and material data, as well as the value of circuit components, are indicated in the figure. The voltage source is a pulse of amplitude $5 \mathrm{~V}$, risetime $200 \mathrm{ps}$, and duration $3 \mathrm{~ns}$. The inverter is realized in CMOS technology, and described via level-2 SPICE MOSFET models. The diode is implemented using a standard library model with a series resistance of $1 \Omega$, a junction capacitance of $2 \mathrm{pF}$, and a saturation current of $50 \mathrm{fA}$. The variability is due to the substrate parameters, i.e. thickness, permittivity and loss tangent, assumed to behave as Gaussian RVs with relative standard deviations of $8 \%, 5 \%$ and $10 \%$, respectively.

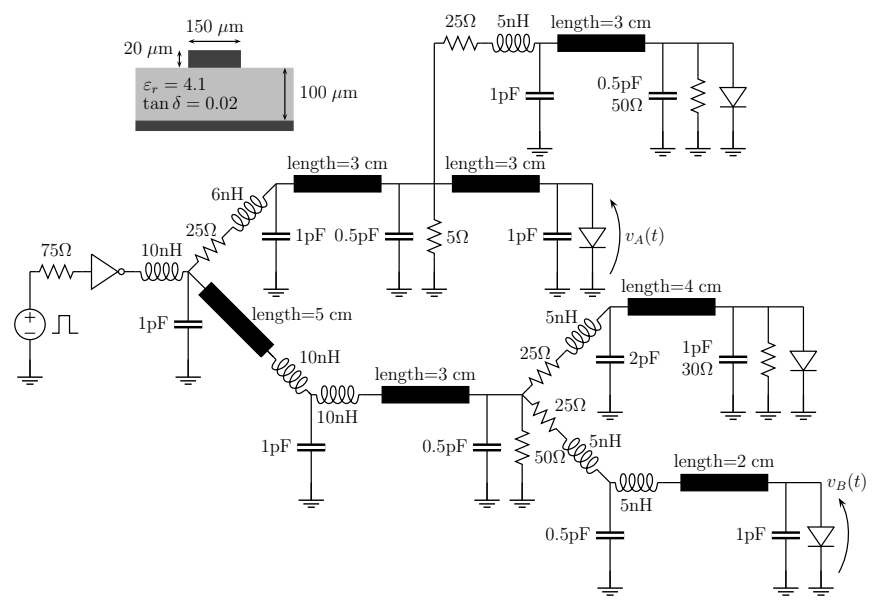

Fig. 4. Interconnect network for the first application example.

Figs. 5 and 6 show two of the voltages appearing at the farend terminations, i.e. $v_{A}(t)$ and $v_{B}(t)$ indicated in Fig. 4. The top panels display the average responses, estimated both from a Monte Carlo analysis (blue line) and with a PC-based simulation (red markers) using $P=9$ and $Q=27$. The gray areas correspond to a small set of random responses and provide a qualitative idea of the spread of the interconnect behavior. The bottom panels compare the results on the estimation of the voltage standard deviations. The good accuracy provided by PC can be appreciated. The Monte Carlo and PC approaches required $2727 \mathrm{~s}$ and $164 \mathrm{~s}$, respectively and therefore, a speedup factor of $17 \times$ is achieved.

\section{B. Example \#2}

The second example concerns the interconnect network depicted in Fig. 7, including three coupled microstrip sections, with the cross-section given at the top-left corner, as well as three inverters and two diodes. These nonlinear components are modeled in HSPICE as in the previous example. The trace width and separation are considered as RVs here, both with a relative standard deviation of $10 \%$. The input voltage source produces a pulse with an amplitude of $5 \mathrm{~V}$, a risetime of $100 \mathrm{ps}$ and a duration of $4 \mathrm{~ns}$.

The statistical properties of the voltage $v_{C}$ at the inverter output are collected in Fig. 8. As in the previous example, average and standard deviation obtained by means of PC (red markers), with $P=5$ and $Q=9$, and the reference 

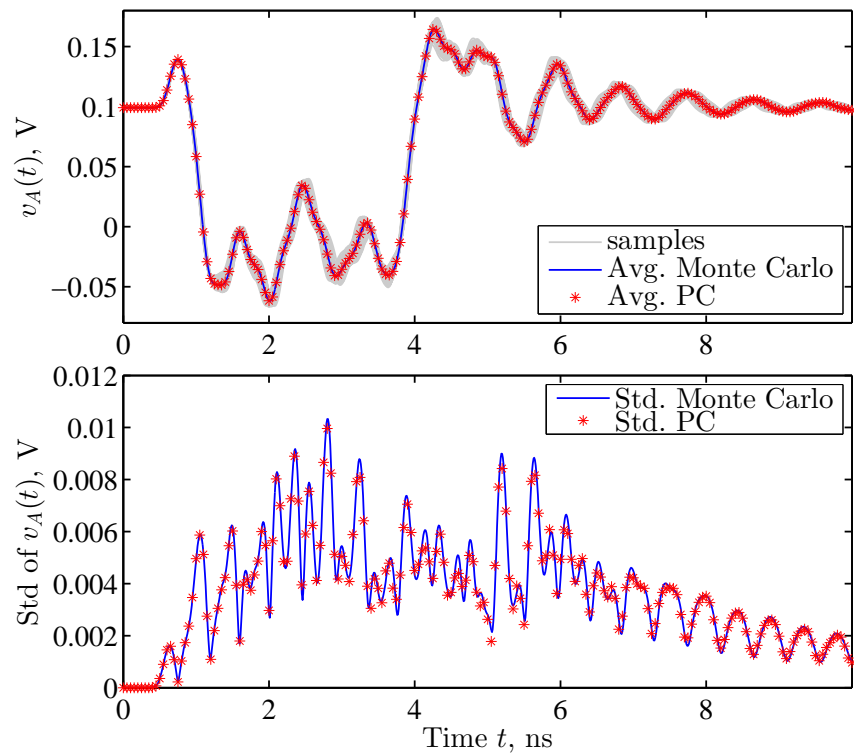

Fig. 5. Statistical assessment of voltage $v_{A}(t)$ in Fig. 4: average (top panel) and standard deviation (bottom panel). Results from Monte Carlo analysis (blue lines) are compared against those obtained with the PC approach (red markers). Some samples of the stochastic voltage are also plotted (gray area) in the top panel and show the response spread.
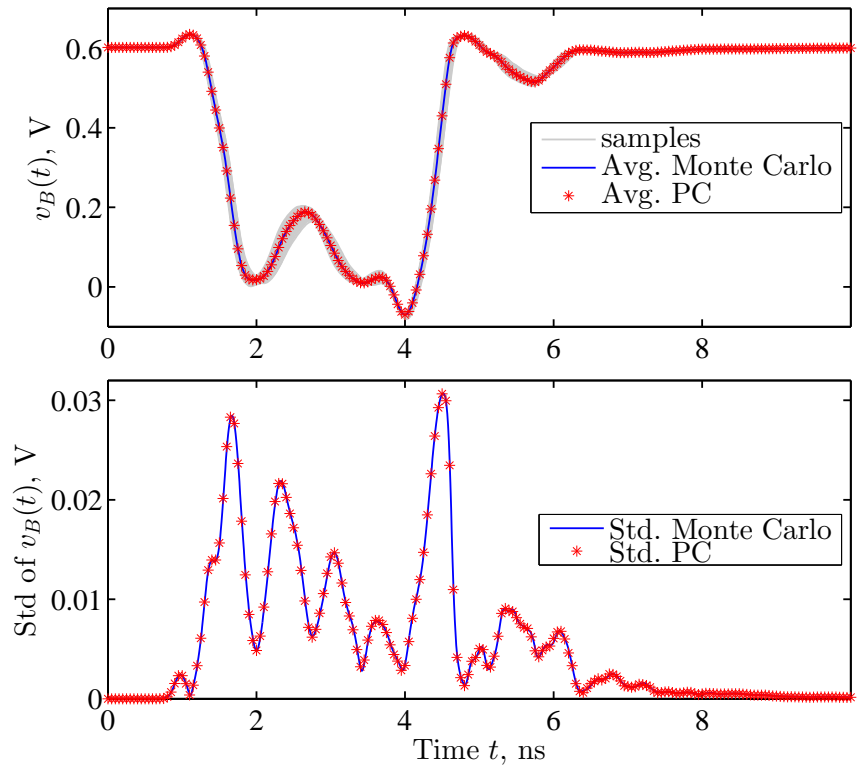

Fig. 6. Statistical assessment of voltage $v_{B}(t)$ in Fig. 4. Curve definition as in Fig. 5.

Monte Carlo method (blue lines) are compared. Analogous information pertaining to voltage $v_{D}$ is reported in Fig. 9. The simulation times are $3087 \mathrm{~s}$ and $66 \mathrm{~s}$ for the Monte Carlo and PC-based simulations, respectively. The speed-up factor for this second example is thus $47 \times$.

Finally, it is worth mentioning that the proposed methodology also allows to easily extract statistical functions such as probability distributions. These are obtained by randomly sampling the PC expansions (2). As an example, Fig. 10

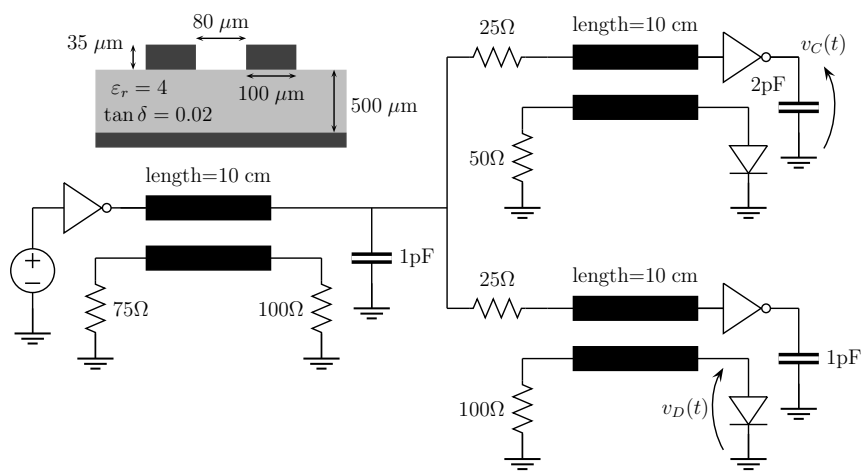

Fig. 7. Interconnect network for the second application example.
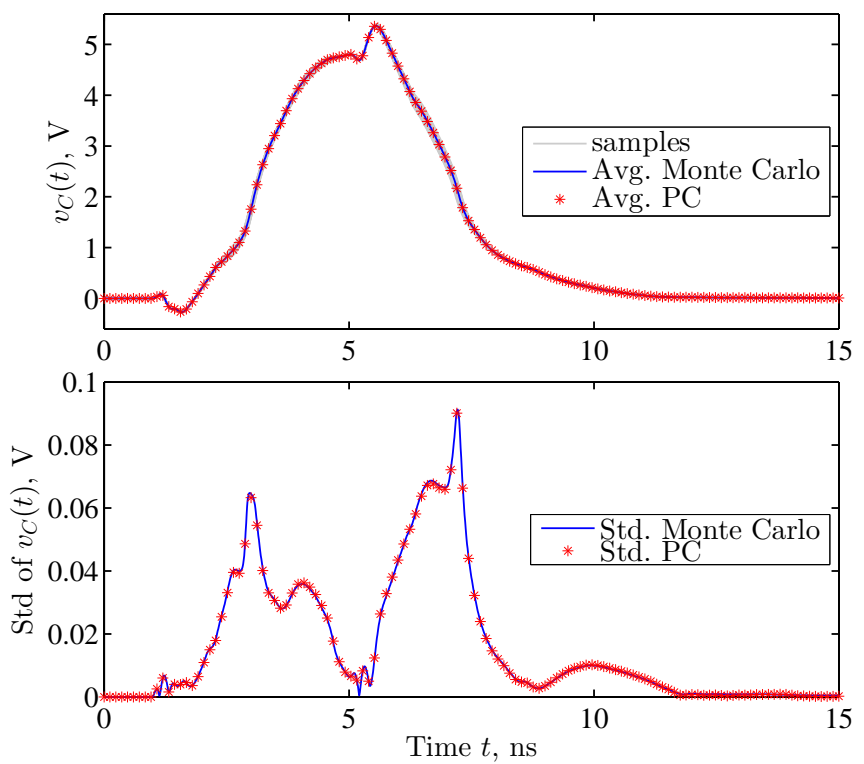

Fig. 8. Statistical assessment of voltage $v_{C}(t)$ in Fig. 7. Curve definition as in Fig. 5.

displays the distribution of voltage $v_{D}$ at $8 \mathrm{~ns}$. The histogram constructed from the collected Monte Carlo samples (gray bars) is compared against the PC estimation (red line). The PC representation (2) allows a better (i.e., smoother) reproduction of the probability density function.

\section{CONCLUSIONS}

This paper addresses the stochastic simulation of lossy, dispersive and distributed interconnect networks that include nonlinear components and that are affected by random crosssectional variations, e.g. due to process tolerances. The advocated technique is based on the PC approach, i.e. on the expansion of circuit variables in term of series of orthonormal polynomials. For this purpose, recent results on the statistical analysis of transmission lines and of nonlinear circuits are 

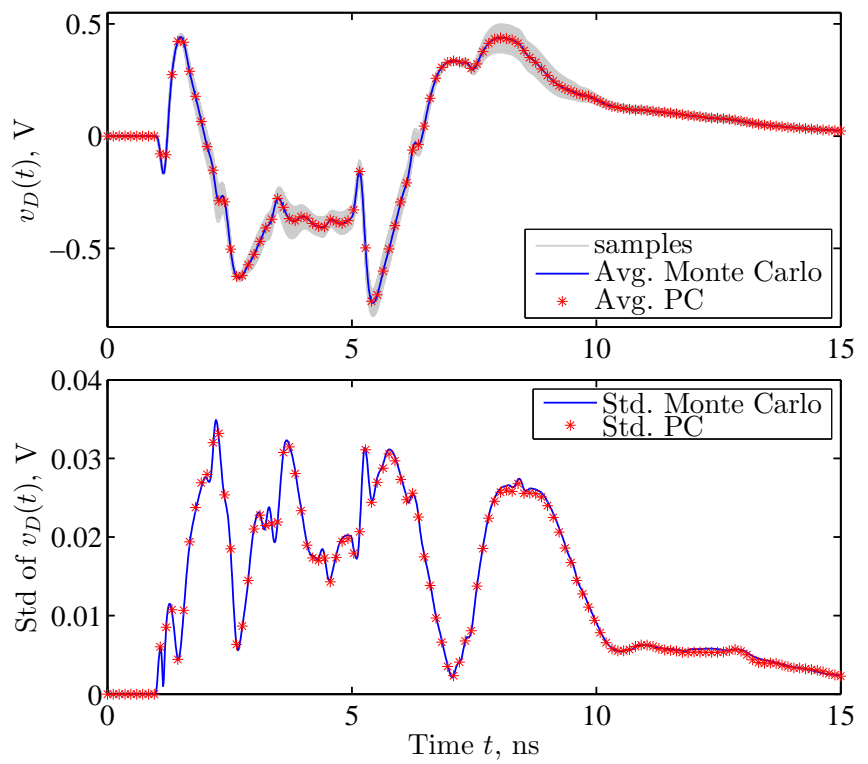

Fig. 9. Statistical assessment of voltage $v_{D}(t)$ in Fig. 7. Curve definition as in with Fig. 5.

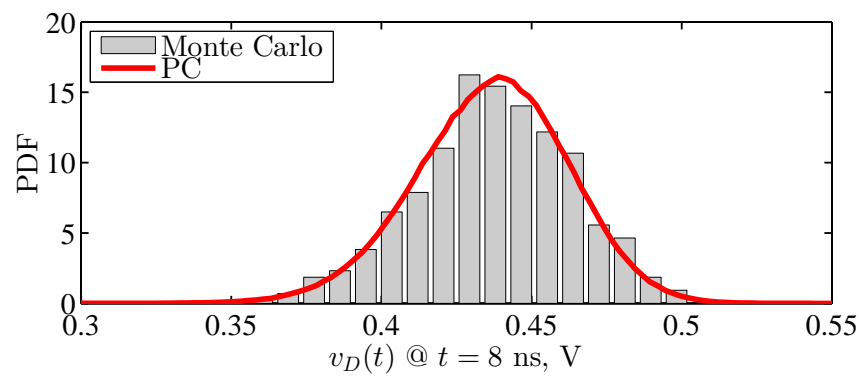

Fig. 10. Probability distribution of $v_{D}(t)$ in Fig. 7 at $t=8$ ns. Gray bars: result from Monte Carlo simulation; red line: PC estimation.

suitably combined.
The PC-expansion coefficients are obtained via a single deterministic simulation of a modified network that includes equivalent circuit representations of the stochastic models. The circuit models are readily implementable in SPICE-type environments, thus allowing designers to take full advantage of well-consolidated algorithms and available device models.

The PC coefficients directly provide pertinent statistical information on the interconnect response such as average, standard deviation and distributions. Remarkable speed-up with respect to Monte Carlo analysis is achieved, since a repeated run is no longer required. Good accuracy is also established. The benefits of the methodology are validated via the analysis of two distributed networks containing inverters and diodes.

\section{REFERENCES}

[1] I. S. Stievano, P. Manfredi, and F. G. Canavero, "Parameters variability effects on multiconductor interconnects via Hermite polynomial chaos," IEEE Trans. Compon. Packag. Manuf. Techol., vol. 1, no. 8, pp. 12341239, Aug. 2011.

[2] P. Manfredi, D. Vande Ginste, D. De Zutter, and F. G. Canavero, "Uncertainty assessment of lossy and dispersive lines in SPICE-type environments," IEEE Trans. Compon. Packag. Manuf. Techol., vol. 3, no. 7, pp. 1252-1258, Jul. 2013.

[3] A. Biondi, D. Vande Ginste, D. De Zutter, P. Manfredi, and F. G. Canavero, "Variability analysis of interconnects terminated by general nonlinear loads," IEEE Trans. Compon. Packag. Manuf. Techol., vol. 3, no. 7, pp. 1244-1251, Jul. 2013.

[4] P. Manfredi, D. Vande Ginste, D. De Zutter, and F. G. Canavero, "Stochastic modeling of nonlinear circuits via SPICE-compatible spectral equivalents," accepted for publication on IEEE Trans. Circuits Syst. I, Reg. Papers.

[5] D. Xiu, "Fast numerical methods for stochastic computations: a review," Communications in Computational Physics, vol. 5, no. 2-4, pp. 242-272, Feb. 2009.

[6] C. R. Paul, Analysis of Multiconductor Transmission Lines. New York: Wiley, 1994.

[7] HSPICE User Guide: Signal Integrity, Version B-2008.09, Synopsys, Inc., Mountain View, CA, USA, Sep. 2008. 